\title{
Editorial
}

\section{Presentation of JCSW Issue 2018/2}

by

Kjersti Ørvig

Associate Professor, PhD

University of Stavanger

kjersti.orvig@uis.no

\section{(c) (i) (2)}

This work is licensed under a Creative Commons Attribution-ShareAlike 4.0 International License. 
We are glad to invite our readers to this year's second issue.

In the November issue of the JCSW, there are three articles, with all the contributions tackling the challenges facing social work in different countries.

Both historical and contemporary research provide examples of how social work continues to colonize and do injustice to indigenous people (Godinet, Arnsberger, Li, \& Kreif, 2010; Lawler, LaPlante, Giger, \& Norris, 2012; Lawrence, 2000). In their article, Conceptualizations of Culture and Ethnicity within Social Work in Two Indigenous Communities: Implications for Culturally Adequate Social Work, Reidunn Håhøy Nygård, Merete Saus and Shanley Swanson Nicolay compare social work in Sami communities within Norway and Native American communities in the state of Montana in the US. The authors investigate the conceptualization of culture and ethnicity, and the implications of these constructions for culturally adequate social work practice. The data is based on focus group interviews and individual interviews with social workers in both Sami communities and Native American communities. The data reveals that social workers in Sápmi conceptualize culture and ethnicity as hybrid and fluid, while the social workers in Native American communities have a more fixed and static conceptualization.

The second article by Yan Zhao and Yu Huang is about the experience of Chinese elder parents who migrate domestically to help their adult children with childcare. An important context is a lack of sufficient institutional support around childcare in contemporary China. The article, Migrating (Grand)Parents, Intergenerational Relationship and Neo-Familism in China, specifically investigates the relationship between the migration of elders and their intergenerational relationship with their adult children, or the second generation. By using a case study in one residential community, the authors argue that the migration of the elder (grand)parents is a particular form of descending/neo-familism. This entails an intergenerational solidarity centred on the needs of the younger generations that builds upon intimacy, with the focus and meaning of the life flow downward to the third generation, as well as entailing aspects of self-salvation (Yan, 2017). 
The last article in this issue is written by Goran Basic, and focuses on successful collaborations in social care practice for a young person in Swedish Juvenile Care. The data is based on an ethnographic study of field notes interpreted as beneficial for the youth. The analysed examples reference a completed appointment for an eye test, a practical realization of active leisure, homework help and an internship placement that works. The findings reveal that trust and motivation are important aspects for a successful collaboration, and the inclusion of less powerful individuals and groups of individuals within a community. 